\title{
Introduction: Carotid endarterectomy versus carotid stenting-A never-ending story
}

Stenosis of the internal carotid artery may be responsible for $10 \%$ to $20 \%$ of all strokes or transient ischemic attacks. It should be borne in mind that stroke is the third leading cause of death and the most common cause of permanent disability in Western countries. Until the introduction of carotid artery stenting (CAS), carotid endarterectomy (CEA) was the only surgical solution to the potential embolic and thrombotic load of the carotid plaque. During the past few years, medical therapy has made enormous progress with the introduction of new drugs and the widespread and more effective control of vascular risk factors [1]. The choice of treatment between CEA, CAS, or medical therapy alone for any individual patient with carotid stenosis remains a controversial issue. However, a simple complete method to independently stratify the periinterventional neurologic outcomes of patients undergoing CAS is still lacking [2-5]. CEA has been used to treat carotid disease for more than 50 years. During this period, CEA was validated by large multicenter randomized clinical trials (RCTs) [6-10] as an effective method for stroke prevention. Indications for CEA have been defined, outcomes have markedly improved, and technical aspects have evolved. Despite the wide acceptance of CEA, the ideal surgical technique to optimize early outcomes and long-term durability of CEA has yet to be determined. Consequently, as a practical matter, most vascular surgeons use a number of technical variations for CEA in their clinical practice and adapt their preference to each particular situation. Conventional CEA, performed through a longitudinal arteriotomy of the internal carotid artery, followed by primary closure or patch angioplasty, is the most frequently employed technique. A few prospective randomized studies have demonstrated that when data on three principal outcomes (ie, perioperative strokes, early carotid occlusion, and restenosis $>50 \%$ ) are pooled, the statistical results strongly favor patch over primary closure [11-17]. Eversion CEA was initially reported by De Bakey et al [13], later described by Etheredge [14], and finally adapted by Raithel and Kasprzak [15] as the simplified version used today, which involves complete oblique transection of the internal carotid artery at the bulb. Due to an oblique rather than a longitudinal arteriotomy, the technique should allow the artery to be less prone to restenosis, particularly when sutures are placed at the widest part of the artery (ie, common carotid artery or the base of carotid bulb). Although it is generally agreed that this technique is safe and effective $[16,17]$, some concerns remains regarding the possibility that it is more technically demanding, especially in the case of a long stenosis plaque or clamping ischemia when a shunt is needed.

In the last several years, evolution in both stents and protection devices, as well as in carotid stenting techniques, resulted in an important reduction in stroke rate in patients undergoing CAS procedures. Carotid stenting, although it is a mature technique, regularly applied, and with excellent outcomes in high-volume centers by expert operators, is struggling to find the consensus of the scientific community. In fact, the initial enthusiasm for CAS as a valuable and lessinvasive alternative to CEA has been mitigated by the undisputable gap in outcomes between the two strategies observed in RCTs. Inadequate requirements in terms of endovascular expertise, potentially leading to an increased event rate related to both insufficient technical skills and inadequate patient selection, has been proposed as the main reason for the unfavorable outcomes related to CAS. Despite the fact that high-quality multicenter registries and high-volume, single-center experiences have consistently described favorable CAS outcomes, the evidence has not been considered sufficient in the neurologist community to recommend CAS. Certainly a key issue to limit the periprocedural events to the lowest possible is to select the appropriate device for the appropriate patient anatomy and clinical syndrome. Several studies have shown that the so-called "tailored approach" for the treatment of carotid disease may offer the best-available results in term of acute and long-term outcomes. The basic principle of the tailored approach [18] is the selection of the appropriate stent and protection device for a specific patient with a specific lesion and vascular anatomy with the flexibility to use the most appropriate devices and techniques to manage each individual patient without limitation of an RCT protocol, such as the restricted use of a particular embolic protection device and stent system. An important element of 
this concept is the recognition of high-risk cases for CAS dependent primarily on the skill of the interventional vascular specialist, a factor that is substantially more relevant in the field of CAS than other areas of percutaneous intervention. In fact, Pieniazek et al [19] demonstrated that tailored CAS is associated with low complication rate and high longterm efficacy, indicating that operators should be familiar with the use of different and multiple protection devices and stent systems in order to be able to use the most appropriate one. Other authors have written about tailored CAS, focusing their attention on the kind of stent and embolic protection device used. Carotid stenting, although it is a mature technique regularly applied with excellent outcomes in highvolume centers by expert operators, is struggling to find the consensus of the scientific community. The initial enthusiasm for CAS as a valuable and less-invasive alternative to CEA has been mitigated by the undisputable gap in outcomes between the two strategies observed in RCTs. But in the last several years, evolution in both the stents and protection devices, as well as in carotid stenting techniques, has resulted in an important reduction in stroke rate in patients undergoing CAS procedures. In fact, because embolization through the stent struts is the primary suspected cause of early postoperative neurologic complications, surgical technology has focused on the production of a new generation of stents with a double layer of mesh to reduce the "free area" of the cells, and new embolic protection devices to reduce the risk of intraprocedural embolization to the brain. So although there is still the need for level I evidence from RCTs testing these new tools, we believe that new materials in the hand of skilled operators who are able to choose the appropriate carotid stent and protection device based on the plaque morphology and patient's anatomical characteristics may definitively fill the gap of CAS in comparison to CEA [20-23]. There is great interest in the possibility of learning additional details regarding the interaction between a carotid plaque and a stent by optical coherence tomography, considering that plaque prolapse through the cell stents has been suggested as one of the major causes of post-procedural complications after CAS, and that available intraprocedural imaging systems (eg, angiography, intravascular ultrasound, and duplex ultrasound) may not be able to detect these intravascular microdefects.

The following key issues have been identified:

1. CAS offers a potential alternative to CEA because it is less invasive, avoids most of the surgical complications, and prevents future stroke events.

2. Data from RCTs demonstrate significant differences between CAS and CEA in terms of early neurologic outcomes (from 0 to 30 days), although mid- and long-term neurologic results are indistinguishable.

3. Microembolization through the stent struts is considered the primary suspected cause of early postoperative neurologic complication (ie, nondisabling stroke).

4. Distal protection with filters or occlusive distal balloons has several restrictions because it requires crossing the stenosis with the closed devices before protection is achieved, which increases the risk for embolization.
5. Proximal embolic protection devices insulate the brain by interrupting or reversing the blood flow at the level of the carotid bifurcation at the time of the procedure. So there is the crossing of the lesion under protected conditions.

6. The double-layer stents or mesh stents are a new class of carotid stents that promise to offer higher scaffolding of carotid plaque in comparison to previous carotid stent designs, avoiding, or at least limiting, plaque prolapse through the cell struts.

7. The basic principle of the tailored approach is the selection of the appropriate stent and protection device for a specific patient with a specific lesion and vascular anatomy.

With recent stent device approvals, the CAS landscape may now shift significantly from the individual practice level to the global market. Examining what brought the field to its current state is crucial for understanding possible future changes. It is in that spirit that this issue of Seminars in Vascular Surgery focuses on revising the status of CAS, highlighting the various state-of-the-art treatment modalities currently available, and the preprocedural and intraprocedural strategies that can assist in improving outcomes and reducing complications.

In the first article of this issue, Alberto Cremonesi et al describe the tips and tricks for a correct CAS procedure, starting from basics and including specific anatomical scenarios and tailored techniques for safe CAS. Next, Peter Schneider and Juan Carlos Parodi describe the importance and proper use of embolic protection devices during CAS, including specific devices, as well as multiple anatomic and clinical factors. A number of suggestions about use in various scenarios and how to improve outcomes are provided. Marc Bosiers and colleagues report the impact on outcomes of different types of carotid stenting, underlying how in the periprocedural phase (Day 0 through Day 30) there is still some room for improvement of CAS outcomes. To complete this special issue, Gianmarco de Donato and colleagues summarize indications and results of an early CAS after onset of neurologic symptoms, considering the new materials (carotid stents and cerebral protection systems) and enhanced techniques available.

\section{R E F E R E N C E S}

[1] Nanna MG, Gomes P, Njoh RF, et al. Carotid artery stenting versus carotid endarterectomy. Postgrad Med J 2016;92(1091): 532-9.

[2] Cremonesi A, Castriota F, Secco GG, et al. Carotid artery stenting: an update. Eur Heart J 2015;36:13-21.

[3] Liu Z, Fu WG, Guo ZY, et al. Update systematic review and metanalysis of randomized clinical trials comparing carotid artery stenting and carotid endarterectomy in the treatment of carotid stenosis. Ann Vasc Surg 2012;26:576-90.

[4] Illuminati G, Ricco JB, Greco C, et al. Systematic preoperative coronary angiography and stenting improves postoperative results of carotid endarterectomy in patients with asymptomatic coronary artery disease: a randomised controlled trial. Eur J Vasc Endovasc Surg 2010;39:139-45.

[5] Illuminati G, Ricco JB2. Long-term results of a randomized controlled trial analyzing the role of systematic pre-operative 
coronary angiography before elective carotid endarterectomy in patients with asymptomatic coronary artery disease. Eur J Vasc Endovasc Surg 2015;50:26.

[6] Eikelboom BC, Ackerstaff RG, Hoeneveld H, et al. Benefits of carotid patching: a randomised study. J Vasc Surg 1988;7:240-7.

[7] Clagett GP, Patterson CB, Fisher DF Jr, et al. Vein patch versus primary closure for carotid endarterectomy. A randomized prospective study in a selected group of patients. J Vasc Surg 1989;9:213-23.

[8] AbuRahma AF, Khan JH, Robinson PA, et al. Prospective randomized trial of carotid endarterectomy with primary closure and patch angioplasty with saphenous vein, jugular vein, and polytetrafluoroethylene: perioperative (30-day) results. J Vasc Surg 1996;24:998-1006; discussion 1006-7.

[9] Archie JP Jr. Prospective randomized trials of carotid endarterectomy with primary closure and patch reconstruction: the problem is power. J Vasc Surg 1997;25:1118-20.

[10] Lord RS, Raj TB, Stary DL, et al. Comparison of saphenous vein patch, polytetrafluoroethylene patch, and direct arteriotomy closure after carotid endarterectomy. Part I. Perioperative results. J Vasc Surg 1989;9:521-9.

[11] Katz D, Snyder SO, Gandhi RH, et al. Long-term follow-up for recurrent stenosis: a prospective randomized study of expanded polytetrafluoroethylene patch angioplasty versus primary closure after carotid endarterectomy. J Vasc Surg 1994;19:198-203; discussion 204-5.

[12] Counsell CE, Salinas R, Naylor R, et al. A systematic review of the randomised trials of carotid patch angioplasty in carotid endarterectomy. Eur J Vasc Endovasc Surg 1997;13:345-54.

[13] De Bakey ME, Crawford ES, Morris GC Jr, et al. Surgical considerations of occlusive disease of the innominate, carotid, subclavian, and vertebral arteries. Ann Surg 1961;154:698-725.

[14] Etheredge SN. A simple technique for carotid endarterectomy. Am J Surg 1970;120:635-40.

[15] Raithel D, Kasprzak PM. The eversion endarterectomy. A new technique. In: Greenhalgh RM, Hollier LH, et al., eds. Surgery of Stroke. London, UK: WB Sunders; 1993:441-71.
[16] Buerger R. Eversion endarterectomy of the carotid bifurcation. In: Veith FJ, ed, Current Critical Problems in Vascular Surgery, Vol 5. St Louis, MO: Quality Medical Publishing; 1993:441-7.

[17] Cao P, Giordano G, De Rango P, et al. Eversion versus conventional carotid endarterectomy: a prospective study. Eur J Vasc Endovasc Surg 1997;14:96-104.

[18] Nikas DN, Kompara G, Reimers B. Carotid stents: which is the best option? J Cardiovasc Surg (Torino) 2011;52:779-779.

[19] Pieniazek P, Musialek P, Ziembicka AK, et al. Carotid artery stenting with patient- and lesion-tailored selection of the neuroprotection system and stent type: early and 5-year results from a prospective academic registry of 535 consecutive procedures (TARGET-CAS). J Endovasc Ther 2008;15:249-62.

[20] Setacci C, de Donato G. Carotid artery stenting renaissance: can tips, tricks and new devices fill the gap? J Cardiovasc Med (Hagerstown) 2016;17:855-6.

[21] Setacci C, Speziale F, de Donato G, et al. IRON-Guard Study Group. Physician-initiated prospective Italian Registry of carotid stenting with the C-Guard mesh-stent: the IRON-Guard registry. Rationale and design. J Cardiovasc Surg (Torino) 2015;56:787-91.

[22] de Donato G, Mazzitelli G, Ruzzi U, et al. Carotid artery stenting renaissance: is it safe and effective using new materials? J Cardiovasc Surg (Torino) 2016;57:769-83.

[23] Setacci C, Mele M, de Donato G, et al. Device selection for carotid stenting: reviewing the evidence. Expert Rev Cardiovasc Ther 2017;15:787-96.

Guest Editors

Carlo Setacci, Gianmarco de Donato Department of Vascular Surgery University of Siena Siena, Italy

Antonio Sterpetti

Department of Vascular Surgery University Rome La Sapienza Rome, Italy 